\title{
A Nonlinear Dynamic Model of the Financial Crises Contagions*
}

\author{
Ke Chen ${ }^{1,2}$, Yirong Ying ${ }^{1}$ \\ ${ }^{1}$ College of Management, Shanghai University, Shanghai, China \\ ${ }^{2}$ College of Finance and Economics, Chongqing Jiaoting University, Chongqing, China \\ E-mail: ckbest@163.com,yrying@staff.shu.edu.cn \\ Received December 19, 2010; revised January 7, 2011; accepted January 10, 2011
}

\begin{abstract}
Employing the Differential Dynamics Method, a nonlinear dynamic model is set up to describe the international financial crises contagion within a short time between two countries. The two countries' control force depending on the timely financial assistance, the positive attitude and actions to rescue other infected countries, and investor confidence aggregation, and the immunity ability of the infected country are considered as the major reasons to drive the nonlinear fluctuations of the stock return rates in both countries during the crisis. According to the Ordinary Differential Equations Qualitative Theory, we found that there are three cases of financial crises contagion within a brief time between two countries: weak contagion with instability but inhibition, contagion with limit and controllable oscillation, and strong contagion without control in a brief time.
\end{abstract}

Keywords: Financial Crises Contagion, Stock Return Rate, Nonlinear Dynamics Model, Limit Cycle

\section{Introduction}

Since 1990s, the international financial crises have frequently broken out, and their contagions also greatly increased. The crises like the infectious diseases often spread quickly from the earliest crises country or region to others after their outbreak. For instance, the 1994 "tequila crisis" in Latin American countries, the 1998 "Russian virus" and the 1997 Southeast Asian financial crises all are the regional financial crises with strong contagion. However, the crisis trigged by 2007 American sub-prime crisis has more strong contagion, and has developed into a global financial crisis. It has leaded some countries into economic crisis and given a profound impact on the order of global economic development. Therefore, we pay particularly our attention to the dynamic transmission problems of the financial crisis within a short time after its outbreak.

Many researchers have been focused on the financial crisis and its contagion test. But they show the different empirical conclusions under the different definitions of financial crisis contagion. Sometimes the different results may be found even under the same definition framework.

* This work has been supported by the Research Fund of Program Foundation of Education Ministry of China (10YJA790233)
For example, the financial crisis contagion is defined as the significantly enhanced correlation among the financial markets after the crisis outbreak. Reference [1-3] find there are the significantly enhanced correlations in crossborder capital markets when they study some major financial crises. But [4] strictly separates the contagion and mutual dependent degree, and taking account of the different conditions variance, they don't find the evidence that the correlations between the various markets are destroyed during those major crises. For the reasons, [5] have pointed out that the correlation coefficient is only used to describe a linear relationship between the two financial markets, and it is not suitable for studying the non-linear changes. Reference [6] also put forward that without considering the conditional heteroskedasticity, the results of correlation test are biased. Furthermore, [7] suggest that these differences in definitions and tests are small, even the same under certain conditions. And after classifying the empirical research methods of financial contagion and analyzing their similarities and differences, they explain that some models reflect all of the information while others take part of the information. Therefore, we follow the strict distinction on the infectious and mutual dependence in [4], but don't distinguish the amount of information in the model. Then we inves- 
tigate the financial crises contagions between two financial markets by analyzing their mutual impact changes within a short time during the crises. Whatever nonlinear effect is between two financial markets, the contagion may occur if those changes are in an unstable state. Otherwise the markets keep the mutual dependence relationship.

As we have the different angle from the previous literature to analyze financial crisis contagion, it may not be rational for us to employ directly those non-linear research methods based on the previous definition. Although those methods can capture more nonlinear characteristics of the financial crises contagions such as the minimum spanning tree method, Copula functions, seemingly unrelated Probit techniques, GARCH model, symbolic time series analysis, dynamic factor model and other methods [8-14], it has been an outstanding issue how to reduce the test errors of financial crisis contagion between two markets. So we try to introduce the differential dynamics methods to set up a non-linear model of the financial crisis contagion between two markets. Additionally, we use the Ordinary Differential Equations Qualitative Theory to describe the mutual impact state between two countries or two financial markets within a short time during the crisis, then to discuss the infectious state or path.

The remainder of this paper is organized as follows. Section 2 focuses on setting to the nonlinear contagion model by using the differential dynamics methods. Section 3 shows the major theorems based on Ordinary Differential Equations Qualitative Theory and their proofs, and suggests the financial crises contagion cases between two markets. The last section summarizes our studies.

\section{Modeling}

The financial crisis contagion firstly affects the financial security of a country. It may lead to the great volatility of the financial asset prices (such as stocks, bonds, currencies, real estate, etc.), deteriorate the operating conditions of the financial institutions, cause the capital flight, decline the foreign exchange reserves, and increase the foreign debt. And one of the first performances during crisis is the great volatility of the stock price matched with time very well, so that it becomes one of the most common means to use the stock prices or the volatility of the stock return rate for analyzing the financial crisis contagion. Thus, we put forward the nonlinear dynamics model of financial crisis contagion between two countries by examining the dynamic change trends of the stock return rate after the crisis outbreak.

On the one hand, observing directly the changes of the stock return rate in the market, we found that when one country has a financial crisis, its stock returns may drop drastically and quickly affect other countries' stock market in a short time. Then there is a crisis contagion among the crisis country and its neighboring countries or countries with a close contact. On the other hand, summarizing the previous studies, we recognize that the degree or efficiency the crisis country affecting on other countries usually depends on the affected country's immunity ability, control force and investor confidence aggregation. The immunity ability is determined by the fundamental factors such as economic strength, economic structure, financial system safety, financial market openness, management level exchange system, etc. The control force depends on the timely financial assistance, the positive attitude and actions to rescue other infected countries. And the growth of investor confidence aggregation comes largely from the increase of first two capabilities. Furthermore, we suggest that before the crisis, one country's average changing rate of stock returns depends on its immunity ability, and reflects the interdependence relationship with the crisis country. However, during the crisis, affected by itself control force and investor confidence aggregation as well as the crisis country's stock return changes, one country's stock returns may move away from its original average rate and have nonlinear fluctuation following the change of the crisis country's stock market in a short time. Meanwhile, the crisis country's stock returns keep dropping sharply within a short time, and also having the nonlinear volatility impacted by the control force and investor confidence aggregation of itself and other countries.

Thus, we assume that the nonlinear function of the stock return rate in two markets can be conducted to reflect the above nonlinear volatility affected by the control force, investor confidence aggregation and other hidden factors in the two countries. And we adopt the power function to describe that the impact on the other countries is far greater than that on the crisis countries. As a preliminary discussion, we constructed a nonlinear dynamic model with a minimum power law, as follows:

$$
\left\{\begin{array}{l}
d r_{1} / d t=a-r_{1} r_{2}^{2} \\
d r_{2} / d t=r_{2}\left(-b+r_{1} r_{2}\right)
\end{array}\right.
$$

where $a, b$ are positive constants, $a$ is the increasing rate of the average stock returns of A country under the normal situation, and $b$ is the decreasing rate of the stock returns of $\mathrm{B}$ country. $r_{1}, r_{2}$ are respectively the stock return rates of $\mathrm{A}$ and $\mathrm{B}$ country. At the dynamic angle, (1) show that:

1) Within a short time after the crisis outbreak, the stock market of A country can't develop with a constant speed $a$ due to the nonlinear effect of B country, the crisis one. Here $r_{2}^{2}$ is similar to the 
variable coefficient of stock returns in A country. When $r_{2}$ is small, the financial crisis has a smaller impact on the average stock returns of A country. But when $r_{2}$ is becoming larger, the impact of financial crisis on the average stock returns of $\mathrm{A}$ country is going to grow up at the square speed of the larger $r_{2}$.

2) At the first period of the crisis, the increase rate of the average stock returns in B country changes with the speed $-b r_{2}$. That means its return rate may decrease gradually in accordance with exponential law. When the contagion develops to a certain extent, this dropping speed becomes $-b+r_{1} r_{2}$ under the inter-effect of the stock returns in two countries. Additionally, there are: a) When B country increases its control force or the others rise up their control force and investor confidence aggregation, the dropping speed of $r_{2}$ may be controlled, namely $r_{1} r_{2}>0$. b) When those measures don't make any sense, the stock returns of B country may have an accelerative decreasing trend, namely $r_{1} r_{2}<0$.

\section{Analysis}

At first, one can easily find the singular point in (1) is $\bar{o}=\left(b^{2} / a, a / b\right)$.

Exchanging $r_{1}$ and $r_{2}$ in (1), and moving the singular point to the origin of coordinate, (1) can be transformed into the following form.

$$
\left\{\begin{array}{l}
d r_{1} / d t=b r_{1}+a^{2} r_{2} / b^{2}+r_{1}\left(b r_{1} / a+2 a r_{2} / b+r_{1} r_{2}\right) \\
d r_{2} / d t=-2 b r_{1}-a^{2} r_{2} / b^{2}-b^{2} r_{1}^{2} / a^{2}+r_{2}\left(r_{1}^{2}+2 a r_{1} / b\right)
\end{array}\right.
$$

According to the Ordinary Differential Equations Qualitative Theory, it is easy to find the conclusions as follow.

Theorem 1 The characteristics of the singular point $\bar{o}=\left(b^{2} / a, a / b\right)$ in (1) are:

1) if $a^{2}>b^{3}, \bar{o}$ is a stable node or focus;

2) if $a^{2}<b^{3}, \bar{o}$ is an unstable node or focus;

3) if $a^{2}=b^{3}, \bar{o}$ is a center point or focus.

Proof: The characteristic equation of the corresponding linear part of (1) at point $\bar{o}$ is:

$$
\lambda^{2}+\left(a^{2} / b^{2}-b\right) \lambda+a^{2} / b^{2}=0
$$

Notice that $p=a^{2} / b^{2}-b$ and $q=a^{2} / b^{2}>0$. Thus, if $b<0, \bar{o}$ is the saddle point. If $p>0$, namely $a^{2}>b^{3}$, $\bar{o}$ is the stable node or focus. And if $p<0$, namely, $\bar{o}$ is the unstable node or focus. And if $p=0$, namely $a^{2}=b^{3}, \bar{o}$ may be a center point or focus.

Lemma Equation (1) may be described as the Lienard Equation. That is:

$$
\left\{\begin{array}{l}
d x / d t=y-F(x) \\
d y / d t=-g(x)
\end{array}\right.
$$

where

$$
\begin{gathered}
F(x)=x+\left(b x+b^{2} x^{2} / a\right) /\left(a^{2} / b^{2}+2 a x / b+x^{2}\right), \\
g(x)=\left(a^{4} x / b\right) /\left(a^{2}+2 a b x+b^{2} x^{2}\right)
\end{gathered}
$$

Proof: Let

$$
x=r_{1}-b^{2} / a, y=r_{2}-a / b
$$

Then it is easy to obtain:

$$
\left\{\begin{array}{l}
d x / d t=-a^{2} x / b^{2}-2 b y-2 a x y / b-b^{2} y^{2} / a-x y^{2} \\
d y / d t=a^{2} x / b^{2}+b y+2 a x y / b+b^{2} y^{2} / a+x y^{2}
\end{array}\right.
$$

Exchanging $x$ and $y$ in (4), and still mark $x$ and $y$, then one can get

$$
\left\{\begin{array}{l}
d x / d t=A_{0}(x)+A_{1}(x) \cdot y \\
d y / d t=B_{0}(x)+B_{1}(x) \cdot y
\end{array}\right.
$$

where $A_{0}(x)=b x+b^{2} x^{2} / a, \quad B_{0}(x)=-2 b x-b^{2} x^{2} / a$

$$
A_{1}(x)=-B_{1}(x)=a^{2} / b^{2}+2 a x / b+x^{2}
$$

Thus it can be proved in accordance with Lemma 6.3 proposed by [15].

Theorem 2 If $b>0$ and $a^{2}=b^{3}$, the singular point of (1) is an unstable focus.

Proof: Introduce the following binary function,

$$
H\left(r_{1}, r_{2}\right)=r_{1}^{2}+r_{1} r_{2}+r_{2}^{2} / 2+\varphi_{3}\left(r_{1}, r_{2}\right)+\varphi_{4}\left(r_{1}, r_{2}\right)
$$

where $\varphi_{3}, \varphi_{4}$ are respectively three homogenous polynomial and four homogenous polynomial. When $c_{0}$ is a sufficient smaller positive number, $H\left(r_{1}, r_{2}\right)=c_{0}$ indicates a cluster of closed curves containing the origin. Considering the differential dynamical system as follow:

$$
\left\{\begin{array}{l}
d r_{1} / d t=b r_{1}+b r_{2}+r_{1}^{2} / b+2 \sqrt{b} r_{1} r_{2}+r_{1} r_{2}^{2} \\
d r_{2} / d t=-2 b r_{1}-b r_{2}+r_{1}^{2} / b-2 \sqrt{b} r_{1} r_{2}-r_{1} r_{2}^{2}
\end{array}\right.
$$

After simple calculation, one can obtain

$$
d H /\left.d t\right|_{(5)}=b\left(r_{1}^{2}+r_{2}^{2}\right)^{2}+h_{5}\left(r_{1}, r_{2}\right)
$$

Here $h_{5}\left(r_{1}, r_{2}\right)$ is a polynomial with less than 5 degree.

Denote $s=\left(2+9 \sqrt{b}+13 b^{3}\right) / 8 b^{4}, t=\left(1+3 b^{3 / 2}\right) / 4 b^{4}$

And give the specific forms of $\varphi_{3}, \varphi_{4}$ in (5) as follow:

$$
\begin{gathered}
\varphi_{3}\left(r_{1}, r_{2}\right)=m_{1} r_{1}^{3}+m_{2} r_{1}^{2} r_{2}+m_{3} r_{1} r_{2}^{2}+m_{4} r_{2}^{3} \\
\varphi_{4}\left(r_{1}, r_{2}\right)=n_{1} r_{1}^{4}+n_{2} r_{1}^{3} r_{2}+n_{3} r_{1}^{2} r_{2}^{2}+n_{4} r_{1} r_{2}^{3}+n_{5} r_{2}^{4}
\end{gathered}
$$

where the coefficients are respectively 


$$
\begin{gathered}
m_{1}=\left(12 b^{3 / 2}+5\right) / 3 b^{2}, m_{2}=\left(6 b^{3 / 2}+3\right) / b^{2}, \\
m_{3}=\left(4 b^{3 / 2}+2\right) / b^{2}, m_{4}=\left(4 b^{3 / 2}+2\right) / 3 b^{2} \\
n_{1}=-1+10 s+8 t, n_{2}=-\frac{5}{2}-20 s+20 t \\
n_{3}=-\frac{9}{4}+18 s+18 t, n_{4}=-4 s+4 t, n_{5}=\frac{5}{16}-s+t
\end{gathered}
$$

Then, due to $b>0$, it can be proved from (5) that the singular point $\bar{o}$ in (1) is an unstable focus.

Theorem 3 If $a^{2} \geq b^{3}$, there is no limit cycle in (1).

Proof: Denote $\lambda(x, y)=y^{2} / 2+G(x)$, where

$$
\begin{aligned}
G(x)= & \int_{0}^{x} g(z) d z=a^{4} / b^{3} \times \ln (a+b x) / a \\
& \quad a^{4} / b^{2} \cdot x /(a+b x) \\
\because x F & (x)=x^{2}+b x^{2} /\left(\frac{a}{b}+x\right)^{2} \cdot(1+b x / a) \\
\therefore & \text { if } x>-a / b, x F(x)>0(x \neq 0)
\end{aligned}
$$

And there is $g(x)=a^{4} / b(a+b x)^{2} \cdot x$.

Then $d \lambda /\left.d t\right|_{(4)}=-g(x) F(x)<0(x \neq 0, x>-a / b)$

So (4) does not possess any limit cycles. Then there is no limit cycle in (1).

Theorem 4 If $a^{2}<b^{3}$, there is an unique limit cycle in (1).

Proof: According to our Lemma and the Theorem 6.6 proposed by [15], one can get

1) There is $a_{01} \alpha_{0}<0$. Then $b^{3}-a^{2}>0$, namely $a^{2}<b^{3}$;

2) There is $\beta_{0}>0$. Then $a^{2}>0$, namely $a \neq 0$;

3) There is $a_{21} \alpha_{4}>0$. Then $b>0$;

4) There is $\beta_{3}>0$. Then $a^{2}+2 b^{3}>0$

Summarizing the above four conclusions, one can find that Theorem 4 is true.

According to Theorem 2-4, we conclude that there are three cases of financial crises contagions between two countries: weak contagion with instability but inhibition, contagion with limit and controllable oscillation, and strong contagion without control in a brief time.

Case 1, when $a^{2}<b^{3} \quad(b>0)$, Theorem 4 indicates that the (1) has unique limit cycle. Its phase plane shows that there occurs an alternating oscillation of stock returns between two countries. However, this oscillation may not enlarge without any limitation due to there exists a limit cycle. The immunity ability and self-repair capacity of the economy system in both two countries may limit the oscillation magnitude within some controllable size, which depends on the size of the limit cycle. So it is a contagion case with limit and controllable oscillation.

Case 2, when $a^{2}=b^{3} \quad(b>0)$, Theorem 2 suggests that the singular point of (1) is not a center but an unstable focus. Due to there is a little difference between center point and focus, it show that during the first period of financial crises contagions, the stock return rates of two countries increase gradually their oscillation magnitude by a imperceptible way. Thus this stage should be the best time to control the financial crises contagions. We call it as the weak contagion with instability but inhibition.

Case 3, when $a^{2} \geq b^{3} \quad(b>0)$, Theorem 3 dominates that there is no limit cycle in (1). In this case, the financial crises contagions have evolved into a disaster and both the two countries have to endure more and more strong impacts. The governments must take the firmest monetary policies and fiscal policies to curb its spread.

\section{Conclusions}

Within a short time after the crisis outbroke, the stock returns of crisis country would be to drop abruptly and cause quickly the stock returns volatility of other countries. Then there is a nonlinear contagion of the financial crisis. To investigate the above phenomenon, we introduce the differential dynamics methods to construct a simple nonlinear dynamic model and derive four theorems in accordance with the qualitative theory of differential equations. Furthermore, based on the discussions about the stability of focus and the existence of limit cycle in the nonlinear volatility equations of stock returns, we analyze the financial crisis contagion situation between two countries during the crisis, and find there are three cases of financial crises contagions within a short time after the crisis outbroke. First one is that if the average increasing rate square of stock returns in the infected country is more than the decreasing rate cube of stock returns in the crisis country, there is no limit cycle in the nonlinear dynamic model of financial crisis contagion. Thus there is a strong contagion between the two countries to be controlled difficultly within a short time. Second one is that if the former is less than the latter, there is a unique stable limit cycle in this model. Thus there is an oscillation contagion between the two countries, whose oscillation magnitude depends on the size of limit cycle. Third one is that if the former equals to the latter, this model has an unstable focus. Thus there is a weak contagion between the two countries. It is easy to be adjusted to the interdependence state before the crisis.

Our analysis results are closer to the actual state of financial crisis contagion within a short term between the two countries. For example, Hong Kong Monetary Authority expected the U. S. subprime mortgage crisis will not create systemic impact on Hong Kong banking system in its paper submitting to Hong Kong SAR Legislative Council in January of 2008. Later this point was also proved. Using Hong Kong's Hang Seng Index and U. S. S \& P500 stock index, we calculated the stock returns by 
the logarithmical return method and their increasing or decreasing rates. Then we found that before the outbroke of the subprime crisis, the average growth rate of Hong Kong stock returns was about 4\% from 2006 to July of 2007; and that the average decreasing rate of U.S. stock returns was $600 \%$ or more from July to August in 2007. It implies there may be " $a^{2}<b^{3}$ " in a shorter time after the subprime crisis outbroke. So the crisis contagion between U. S. and Hong Kong could be controlled by taking some measurements even if the prices of Asia-Pacific stock markets greatly dropped down in August of 2007.

It indicates that the differential dynamics method is a better way to investigate the financial crisis contagion state or path between the two countries. However, as a preliminary discussion, this paper introduced a power function with the lowest power times into a simple nonlinear dynamic model of crisis contagion. The simple form may be extended to others for the further study of variable contagions among three or more countries. In addition, another further work may be doing some empirical research such as discussing the calculating methods of those two indicators in our simple nonlinear model, testing the contagion states by employing the time series data of stock return, trying to contract some early-warning indicators to monitor timely the volatility of financial markets, and so on.

\section{References}

[1] M. A. King and S. Wadhwani, "Transmission of Volatility between Stock Markets," The Review of Financial Studies, Vol. 3, No. 1, 1990, pp. 5-33. doi: $10.1093 / \mathrm{rfs} / 3.1 .5$

[2] S. Calvo and C. Reinhart, "Capital Flows to Latin America: Is There Evidence of Contagion Effects?” WPS 1619, The World Bank, 1996.

[3] T. Baig and I. Goldfaijn, "Financial Market Contagion in the Asian Crisis," Working Paper No. 98/155, IMF, 1998.

[4] K. J. Forbes and R. Rigobon, "No Contagion, Only Interdependence: Measuring Stock Markets Co-Movements," Journal of Finance, Vol. 57, No. 5, 2002, pp. 2223-2261. doi:10.1111/0022-1082.00494
[5] P. Embrechts, A. J. McNeil and D. Straumann, "Correlation and Dependence in Risk Management: Properties and Pitfalls," In: M. Dempster, Ed., Risk Management: Value at Risk and Beyond, Cambridge University Press, 1999, pp. 176-223.

[6] B. H. Boyer, M. S. Gibson and M. Loretan, "Pitfalls in Tests for Changes in Correlations," Provided by Board of Governors of the Federal Reserve System (U. S.) in Its Series International Finance Discussion Papers No. 597, 1999.

[7] M. Dunger, R. Fry, B. Gonzalez-Hermosillo and V. Martin, "Empirical Modeling of Contagion: A Review of Methodologies," Working Paper No. 04/78, IFM, 2004.

[8] G. J. Ortega and D. Matesanz, "Cross-Country Hierarchical Structure and Currency Crises," International Journal of Modern Physics C, Vol. 17, No. 3, 2006, pp. 333-341. doi:10.1142/S012918310600856X

[9] J. C. Rodriguez, "Measuring Financial Contagion: A Copula Approach," Journal of Empirical Finance, Vol. 14, No. 3, 2007, pp. 401-423. doi:10.1016/j.jempfin.2006.07.002

[10] G. Fazio, "Extreme Interdependence and Extreme Contagion between Emerging Markets," Journal of International Money and Finance, Vol. 26, No. 8, 2007, pp. 1261-1291. doi:10.1016/j.jimonfin.2007.06.006

[11] S. Rim, "Empirical Study on the Impacts of Major Events on Inter-Market Relationships in Asia," Global Business and Finance Review, Vol. 12, No. 2, 2007, pp. 75-87.

[12] S. Rim, "Studies on the Financial Market Integration and Financial Efficiency: Evidences from Asian Markets," The Business Review, Vol. 10, No. 2, 2008, pp. 357-363.

[13] J. G. Brida, D. M. Gómez and W. A. Risso, "Symbolic Hierarchical Analysis in Currency Markets: An Application to Contagion in Currency Crises," Expert Systems with Applications, Vol. 36, No. 4, 2009, pp. 7721-7728. doi:10.1016/j.eswa.2008.09.038

[14] A. Cipollini and G. Kapetanios, "Forecasting Financial Crises and Contagion in Asia Using Dynamic Factor Analysis," Journal of Empirical Finance, Vol. 16, No. 2, 2009, pp. 188-200. doi:10.1016/j.jempfin.2008.10.004

[15] L. Li, Y. Ying and X. Dang, "The Significance, Methodology and Application about Infinite," Press of Northwestern University, Xi'an, 1996. [in Chinese] 